\title{
The profile of musculoskeletal pain and its associations with sleep quality and depression during the COVID-19 in Turkey
}

\author{
Merve Karatel, Zeynep Irem Bulut, Erkin Oguz Sari, Zerrin Pelin, and Yavuz Yakut \\ Department of Physiotherapy and Rehabilitation, Faculty of Health Sciences, Hasan Kalyoncu University, Gaziantep, Turkey
}

Received July 5, 2021

Revised August 30, 2021

Accepted September 10, 2021

Handling Editor: Jeong-Gill Leem

\section{Correspondence}

Merve Karatel

Department of Physiotherapy and

Rehabilitation, Faculty of Health

Sciences, Hasan Kalyoncu University, on

Airport Road 8. km, Gaziantep 27010,

Turkey

Tel: +903422118080

Fax: +903422118081

E-mail: kmerve.karatel@hku.edu.tr
Background: The current pandemic has affected people's health multidimensionally. This study aims to investigate musculoskeletal pain, sleep quality, depression levels, and their relationships in individuals belonging to different age groups during COVID-19 in Turkey.

Methods: A web-based self-administered survey that consisted of demographic questions, The Beck Depression Inventory (BDI), Pittsburgh Sleep Quality Index (PSQI), and scores of musculoskeletal pain was sent to participants. Pearson correlation analysis was used for determining the statistical relationship between variables.

Results: The study includes 1,778 participants. The highest percentage for mild, moderate, and severe pain was in the head $(49.8 \%)$, back (15.5\%), and head (11.5\%), respectively. The PSQI-total had shown a weak correlation with pain levels in all body parts. The highest correlation for sleep quality and pain levels was between the PSQI-5 and lower back pain. There was a weak correlation between PSQI2 and the BDI score, and a moderate correlation between the PSQI-1, PSQI-5, PSQI7, PSQI-total, and BDI score. Pain in all body parts showed a weak correlation with depression level.

Conclusions: This study showed that musculoskeletal pain was varied in body parts with different intensities according to age groups in Turkey during the pandemic. The most common pain was in the head, back, and lower back. Headache was found correlated with the parameters of sleep quality. Pain of the head, neck, back, lower back, and shoulder were correlated with sleep latency. Sleep quality was associated with depression and musculoskeletal pain, while musculoskeletal pain was correlated with depression.

Key Words: COVID-19; Depression; Headache; Low Back Pain; Musculoskeletal Pain; Pain; Pandemics; Sleep; Surveys and Questionnaires.

\section{INTRODUCTION}

COVID-19 began to appear on December 19, 2019 and was declared a pandemic by the World Health Organization (WHO) on March 11. COVID-19 began to be seen in Turkey starting in March. According to data from the WHO dated
April 27, 2021, COVID-19 has infected more than 3.3 million people and caused more than 245,000 deaths [1].

In line with these developments, restrictions began to be imposed by governments and the normal lifestyle has changed. With these changes, mandatory social isolation was implemented within the scope of quarantine. (a) This is an open-access article distributed under the terms of the Creative Commons Attribution Non-Commercial License (http://creativecommons.org/licenses/by-nc/4.0/), which permits unrestricted non-commercial use, distribution, and reproduction in any medium, provided the original work is properly cited.

(C) The Korean Pain Society, 2022
Author contributions: Merve Karatel: Writing/manuscript preparation; Zeynep Irem Bulut: Writing/manuscript preparation; Erkin Oguz Sari: Writing/manuscript preparation; Zerrin Pelin: Writing/manuscript preparation; Yavuz Yakut: Formal analysis. 
Staying at home is the most important strategy to reduce COVID-19 contagiousness, control infection, and reduce mortality [2]. In order to prevent the epidemic, students were directed to distance education and employees started working from home. With social isolation, we entered a period where no one goes out unless they have to [3]. Due to the restrictions in dealing with the pandemic, more use of digital devices and reduced physical activity pose a threat to sleep patterns and mental health. People go to bed late at night compared to before the lockdown period, and wake up later in the morning. It is observed that sleep quality is negatively affected in young adults who use digital devices, especially close to bedtime [4].

The widespread use of social media, which increases digital use, is associated with the increase in the tendency to develop sleep problems and a disruption of daily activities [5]. Physical inactivity can cause a decrease in sleep quality [6]. In a study by Majumdar et al. [4], a significant portion of the individuals stated that they felt sleepy at an unusual hour of the day and that their sleep was probably affected by mental stress, anxiety, and exposure to the screen before bedtime.

Sleep is critical for physical health and the effective functioning of the immune system [4]. Sleep disturbances can disrupt the basic processes that contribute to the development and maintenance of joint pain [7]. There is a bidirectional relationship between sleep and pain $[7,8]$. As pain can disrupt sleep, short or inefficient sleep also lowers pain thresholds and increases spontaneous pain. There is neurobiological evidence of mechanisms thought to play a role in the modulation of pain with sleep deprivation. Due to sleep disorders, hyperalgesia and development or exacerbation of spontaneous pain symptoms may cause. This bidirectional relationship between lack of sleep and pain may cause sleep deprivation and a vicious cycle of pain in individuals with chronic pain [8]. In the relationship between sleep and pain, there are biopsychosocial variables such as positive and negative emotion, brain dopamine and opioid systems, age, ethnicity, and gender affect. In the relationship between sleep and pain, attention should be paid to biological, psychological, and social possibilities [7].

In the pandemic period when individuals cannot leave their homes, the need to perform physical activity in a limited area and psychosocial factors are factors in the emergence of pain [9]. During the pandemic process, the human being a biopsychosocial presence is affected in all aspects. Less active lifestyle habits develop during the pandemic process, which increases physical inactivity due to the restrictions. Increased physical inactivity, sleep problems [10], fatigue symptoms [11], weight gain, psychological disorders, and musculoskeletal pain [12] have started to increase. There is a relationship between lifestyle habits of reduced activity and musculoskeletal pain [13]. As the stressful environment increases anxiety levels due to the pandemic, physical and mental health is affected $[9,14]$.

Musculoskeletal problems are a condition that affects millions of office workers, and the increase in working in non-ergonomic environments compounds musculoskeletal problems [9]. With the COVID-19 outbreak, reduced physical activity levels and increased sitting time have been associated with chronic low back pain. Chronic low back pain was more common in individuals who were between the ages of 35-49, had a body mass index of 30 and above, did not comply with ergonomic recommendations, sat for prolonged periods, had inadequate physical activity, or were engaged in remote work or distance education [15]. In addition, with psychosocial influences, the severity of pain increased in the quarantine process for individuals with chronic pain [16]. In a study investigating those who work from home and those who continue to work outside the home, it was observed that low back pain, coronaphobia, and musculoskeletal system complaints were more common in home workers [6].

The pandemic period affects people with its psychosocial dimension as well as physically. During the pandemic process, chronic pain can be triggered by the exacerbation of pre-existing physical and mental complaints as a result of virus damage and the increase in risk factors (poor sleep, inactivity, fear, anxiety, and depression) [17]. Chronic pain is considered in the context of a biopsychosocial model that sees symptoms as the result of a complex and dynamic interaction between biological, psychological, and social factors $[17,18]$. There is a relationship between anxiety, stress, and the inability to cope adequately with these difficult situations and pain $[19,20]$. Musculoskeletal pain is among the most common complaints encountered during routine medical practices, and pain management is an important issue due to the lack of access to medical resources during the process of COVID-19 [21].

Although there are studies in the literature that examine parameters such as pain, sleep, and depression, there are no studies that give all of them in one study and have a large number of individuals participating in the study. The aim of this study was to investigate the relationships between musculoskeletal pain, sleep quality and depression levels in individuals belonging to different age groups. 


\section{MATERIALS AND METHODS}

\section{Participants}

The study included 1,778 individuals from Turkey. The individuals' age was 18-64 years. The participants consisted of academicians, students, health-workers, outside workers, and nonemployees. Individuals outside the age of 18-64 were excluded from the study because they had curfews due to age restrictions and, also individuals who self-quarantined due COVID-19 infections were excluded. In addition, the individuals who had chronic diseases or any other medical conditions were excluded from the study, due to possible sensitivity to musculoskeletal pain or chronic pain which are the primary outcomes of this study.

The University Ethics Committee approved the study protocol (2020/037 on May 28, 2020). Participation in the study was voluntary, and participants were informed about the study. Before the participation, informed consent was obtained from each individual.

\section{Measures and procedures}

This study was a web-based cross-sectional study. From May 29 to July 13, 2020, an online questionnaire link was sent to participants via social networks. Participants submitted their responses anonymously. The questionnaire consisted of general demographic questions, questions from The Beck Depression Inventory (BDI) and The Pittsburgh Sleep Quality Index (PSQI), and musculoskeletal pain scores.

The BDI was used for evaluating the severity of depression in the participants in this study. This questionnaire was comprised of 21 questions, and it was a self-report inventory. The maximum score on the scale was 63 and a higher BDI score specifies higher depression [22]. The PSQI was used to assess participants' sleep quality in this study. The PSQI is valid and reliable in evaluating the quantitative sleep status for the Turkish population [23]. It consists of 24 questions in 7 subscales as follows: subjective sleep quality, sleep latency, sleep duration, habitual sleep activity, sleep disturbances, use of sleep medication, and daytime dysfunction. Higher PSQI scores indicate poor quality of sleep [24].

The visual analogue scale (VAS) was used to score musculoskeletal pain experienced by individuals for at least one month during the study period. The following body parts' pain was assessed using the VAS: the head, neck, back, lower back, shoulder, hand, hip, knee, and foot. The pain scores between 0-3 were included in the mild pain group, those between 4-6 were included in the moderate pain group, and severe pain group included those scoring between 7-10 [25].

\section{Statistical analysis}

The statistical analysis was performed using SPSS version 23 (SPSS Inc, Armonk, NY) software. Data were expressed as mean \pm standard deviation and percentage. The alpha level was 0.05 for all tests of statistical significance. Due to the data being normally distributed, the Pearson correlation analysis was used to measure the statistical relationship between variables. The correlation cofficients which were $\leq 0.35$ were evaluated as weak correlations, 0.36 to 0.67 as moderate correlations, and 0.68 to 1.0 as strong correlations [26]. Also, in some parameters there were missing data due to some participants' unresponsive or inappropriate responses and they were removed. The missing data are specified in the relevant table.

\section{RESULTS}

\section{Demographics of the participants}

The study includes 1,778 responses of $1,131(63.6 \%)$ women and $647(36.4 \%)$ men. Mean age was $28.3 \pm 10.7$ years and mean body mass index was $23.8 \pm 4.4 \mathrm{~kg} / \mathrm{m}^{2}$.

\section{Distribution of pain severity in body parts}

In Table 1, pain severity in body parts is detailed by giving the percantages reported by participants. The highest percantage for mild levels of pain was $49.8 \%$ in the head; the highest moderate pain level was $15.5 \%$ in the back; and highest severe level of pain was $11.5 \%$, also in the head. Table 2 explores percantages of pain levels in body parts according to age group, comparing those 18-34, 35-49, and 50-64 years. There was found to be no relationship between ages and pain level in any body part.

\section{Relationship of sleep, depression and pain severity in body parts}

The relationship of PSQI components and of pain levels in body parts is given in Table 3 according to Pearson correlation analysis. PSQI 3, PSQI 4, and PSQI 6 did not have a association $(0.00<\mathrm{r}<0.10, P<0.001)$., or showed weak correlation with pain in body parts $(0.00<\mathrm{r}<0.20, P<$ 0.001). The PSQI total had shown a weak correlation with pain levels in all body parts, and a correlation coefficient ranged from 0.226 to $0.337(P<0.001)$. The highest correlation for sleep quality and pain levels in body parts was 
Table 1. Distribution of pain severity in body parts $(n=1,778)$

\begin{tabular}{lcccc}
\hline Body parts & None & Mild & Moderate & Severe \\
\hline Head & $420(23.6)$ & $884(49.7)$ & $269(15.1)$ & $205(11.5)$ \\
Neck & $658(37.0)$ & $705(39.7)$ & $241(13.6)$ & $174(9.8)$ \\
Back & $591(33.2)$ & $711(40.0)$ & $275(15.5)$ & $201(11.3)$ \\
Lower back & $648(36.4)$ & $684(38.5)$ & $256(14.4)$ & $190(10.7)$ \\
Shoulder & $868(48.8)$ & $566(31.8)$ & $198(11.1)$ & $146(8.2)$ \\
Hand & $1,215(68.3)$ & $418(23.5)$ & $89(5.0)$ & $56(3.1)$ \\
Hip & $1,201(67.5)$ & $407(22.9)$ & $98(5.5)$ & $72(4.0)$ \\
Knee & $1,068(60.1)$ & $475(26.7)$ & $133(7.5)$ & $102(5.7)$ \\
Foot & $1,073(60.3)$ & $478(26.9)$ & $116(6.5)$ & $111(6.2)$ \\
\hline
\end{tabular}

Values are presented as number (\%).

Table 2. Percantages of pain levels in body parts according to age groups

\begin{tabular}{|c|c|c|c|c|c|c|c|c|c|c|}
\hline Age group (yr) & Pain level & Head & Neck & Back & Lower back & Shoulder & Hand & Hip & Knee & Foot \\
\hline \multirow[t]{4}{*}{$18-34$} & None & $\% 21.2$ & $\% 35.6$ & $\% 31.7$ & $\% 34.5$ & $\% 47.7$ & $\% 68.5$ & $\% 67.8$ & $\% 60.9$ & $\% 62.4$ \\
\hline & Mild & $\% 50.4$ & \%39.8 & $\% 40.0$ & \%39.1 & $\% 32.2$ & $\% 23.3$ & $\% 22.7$ & $\% 26.5$ & $\% 25.6$ \\
\hline & Moderate & \%15.8 & $\% 14.0$ & $\% 16.2$ & \%15.1 & \%11.3 & $\% 5.1$ & $\% 5.6$ & $\% 7.5$ & $\% 6.1$ \\
\hline & Severe & $\% 12.6$ & \%10.6 & \%12.1 & \%11.3 & $\% 8.7$ & \%3.1 & \%3.9 & $\% 5.1$ & $\% 5.9$ \\
\hline \multirow[t]{4}{*}{$35-49$} & None & $\% 27.9$ & \%33.3 & $\% 34.0$ & $\% 37.4$ & $\% 46.1$ & $\% 66.3$ & $\% 66.7$ & $\% 61.3$ & $\% 54.2$ \\
\hline & Mild & $\% 45.5$ & \%39.1 & $\% 37.7$ & $\% 34.3$ & \%31.6 & $\% 24.2$ & \%21.9 & $\% 22.2$ & $\% 27.6$ \\
\hline & Moderate & \%14.5 & \%17.5 & $\% 17.2$ & \%15.8 & \%12.5 & $\% 5.7$ & $\% 6.7$ & $\% 8.4$ & $\% 8.4$ \\
\hline & Severe & $\% 12.1$ & \%10.1 & \%11.1 & \%12.5 & $\% 9.8$ & $\% 3.7$ & $\% 4.7$ & \%8.1 & $\% 9.8$ \\
\hline \multirow[t]{4}{*}{$50-64$} & None & $\% 40.7$ & $\% 50.0$ & $\% 45.8$ & $\% 51.7$ & $\% 59.3$ & $\% 71.2$ & $\% 67.8$ & $\% 50.8$ & $\% 61.0$ \\
\hline & Mild & $\% 51.7$ & $\% 43.2$ & $\% 44.1$ & \%37.3 & $\% 31.4$ & $\% 22.0$ & \%24.6 & $\% 37.3$ & $\% 29.7$ \\
\hline & Moderate & $\% 5.9$ & $\% 2.5$ & $\% 4.2$ & $\% 5.1$ & $\% 5.1$ & $\% 3.4$ & $\% 2.5$ & \%5.1 & $\% 4.2$ \\
\hline & Severe & \%1.7 & \%4.2 & \%5.9 & \%5.9 & $\% 4.2$ & \%3.4 & \%5.1 & \%6.8 & \%5.1 \\
\hline
\end{tabular}

Table 3. Relationship of pain levels in body parts and Pittsburgh Sleep Quality Index (PSQI)

\begin{tabular}{|c|c|c|c|c|c|c|c|c|c|c|}
\hline PSQI parameters & & Head pain & Neck pain & Back pain & $\begin{array}{l}\text { Lower back } \\
\text { pain }\end{array}$ & $\begin{array}{c}\text { Shoulder } \\
\text { pain }\end{array}$ & Hand pain & Hip pain & Knee pain & Foot pain \\
\hline PSQI $1(n=1,778)$ & $r$ & $0.267 * * *$ & $0.268 * * *$ & $0.301 * * *$ & $0.303 * * *$ & $0.247 * * *$ & $0.194 * * *$ & $0.230 * * *$ & $0.190 * * *$ & $0.214 * * *$ \\
\hline PSQI $2(n=1,777)$ & $r$ & $0.264 * * *$ & $0.239 * * *$ & $0.244 * * *$ & $0.219 * * *$ & $0.216 * * *$ & $0.161 * * *$ & $0.143 * * *$ & $0.121 * * *$ & $0.159 * * *$ \\
\hline PSQI $5(n=1,774)$ & $r$ & $0.330 * * *$ & $0.312 * * *$ & $0.340 * * *$ & $0.351 * * *$ & $0.318 * * *$ & $0.261 * * *$ & $0.296 * * *$ & $0.282 * * *$ & $0.310 * * *$ \\
\hline PSQI $7(n=1,778)$ & r & $0.272 * * *$ & $0.295 * * *$ & $0.308 * * *$ & $0.299 * * *$ & $0.270 * * *$ & $0.214 * * *$ & $0.260 * * *$ & $0.209 * * *$ & $0.214 * * *$ \\
\hline PSQI total $(n=1,712)$ & r & $0.312 * * *$ & $0.306 * * *$ & $0.337 * * *$ & $0.326 * * *$ & $0.288 * * *$ & $0.226 * * *$ & $0.284 * * *$ & $0.249 * * *$ & $0.268 * * *$ \\
\hline
\end{tabular}

r: correlation coefficient.

$* * * P<0.001$.

between PSQI 5 and lower back pain levels $(\mathrm{r}=0.351, P<$ $0.001)$. Due to missing data, the sample size was specified for all parameters.

When examining the relationship of depression level and sleep quality; there was a weak correlation between PSQI 2 and the BDI score ( $\mathrm{r}=0.277, P<0.001)$, and a moderate correlation between PSQI 1, PSQI 5, PSQI 7, the PSQI total, and the BDI score $(\mathrm{r}=0.477,0.416,0.441,0.457, P<$ 0.001). Depression level had no association with PSQI 3, PSQI 4, or PSQI 6 ( $\mathrm{r}=0.060,0.101,0.130, P<0.001$ ).

Table 4 outlines the relationship of depression level and pain location according to Pearson correlation analysis. There was statistical relationship found between pain location and BDI scores $(P<0.001)$. Pain in all body parts showed weak correlation with depression level.

\section{DISCUSSION}

The aims of this study are to investigate the profile of musculoskeletal pain of the Turkish population and its associations with sleep quality and depression during the CO- 
Table 4. Relationship of depression level and pain location

\begin{tabular}{lccccccccc}
\hline Beck Depression Scale & Head pain & Neck pain & Back pain & $\begin{array}{c}\text { Lower back } \\
\text { pain }\end{array}$ & $\begin{array}{c}\text { Shoulder } \\
\text { pain }\end{array}$ & Hand pain & Hip pain & Knee pain Foot pain \\
\hline Correlation coefficient $(r)$ & $0.334 * * *$ & $0.292 * * *$ & $0.323 * * *$ & $0.309 * * *$ & $0.299 * * *$ & $0.264 * * *$ & $0.258 * * *$ & $0.212 * * *$ & $0.231 * * *$ \\
\hline
\end{tabular}

$* * * P<0.001$

VID-19 pandemic. The hypotheses of the study are: During the COVID-19 lockdown (a) Individuals have musculoskeletal pain in different body parts, especially the head, back, and neck region because of sedentary behavior. (b) Sleep quality decreases with increasing musculoskeletal pain in these body parts. (c) Sleep quality decreases with increasing depression. (d) Musculoskeletal pain in these body parts increases with increasing depression. The results showed individuals had severe head, back, and lower back pain; moderate back, head, and lower back pain; and mild head, back, and neck pain during the COVID-19 lockdown. Sleep quality was associated with pain in various body parts and depression. Further, pain in these body parts was found to be correlated with depression.

The present study showed that during the COVID-19 lockdown, individuals experienced musculoskeletal pain in various body parts and at different intensities in Turkey. The most common body parts that experienced pain were the head, back, and lower back with different intensities. Amro et al. [27] found that time using social media, using a computer, laptop, or mobile phone, and musculoskeletal disorders were increasing during the COVID-19 quarantine among university students. Also, they showed that there was a correlation between the severity of pain from musculoskeletal disorders, and time of social media usage [27]. Bontrup et al. [28] showed that sitting behavior is associated with chronic low back pain among office workers. During COVID-19 lockdown, along with increased sedentary behavior and technology usage, musculoskeletal pain may have increased among individuals in Turkey. Also, Toksoy et al. [29] mentioned headache related to face masks in healthcare workers. Similarly, headaches may have been triggered by using the face mask during the pandemic.

Although there was no statistically significant difference, the pain level in most body parts was lower in participants over 50 years of age than the other age groups except for mild pain. This study also found that mild back and knee pain percentages were higher in the 50-64 age group than the other age groups. In addition, according to the results of the study, percentages of no pain in most of the body parts were higher in the subjects aged over 50 years than in other age groups. It was shown that most chronic pain conditions occur in the older population, such as musculoskeletal pain of the low back, neck, and joint pain in these studies [30,31]. The 50-64 age group in our study population may have been more vulnerable to musculoskeletal pain because of its nature. The fact that mild back and knee pain was more common in the participants aged 50-64 may be consistent with the natural process of old age.

However, the percentage of those with no pain in most of the body parts was higher in participants in the 50-64 age group than in other age groups, which seems contradictory this idea. These contradictory results in our study can be attributed to the pandemic process, because participants aged 18-50 tried to continue meeting the requirements of their school or work during the pandemic. This circumstance caused many changes such as working hours and conditions, use of technology, and lifestyle. Certainly, the pandemic process also caused lifestyle changes in individuals over 50 years of age, but we interpreted these results as perhaps being due to the effect of more change in work and school conditions for the younger population.

In recent years, the focus has been on the biopsychosocial model in the approach to headache and other types of pain [32]. Negative mental health outcomes were found, such as stress, anxiety, or depression, in COVID-19 studies [33-35]. In a review, psychological factors were investigated for neck and back pain and it was found that stress, distress, or anxiety were related to neck or back pain [36]. Because of the biopsychosocial nature of the pain, the musculoskeletal pain that occurred in individuals during the COVID-19 lockdown may have resulted from one or many of these factors.

This study found that during the COVID-19 lockdown, sleep quality was associated with pain in certain body parts. The sleep quality score was positively correlated with musculoskeletal pain. The parameters of the PSQI have differed in relationship with pain locations in the body. Especially, sub-parameters of sleep disturbances and daytime dysfunction were related to all body part pain in individuals. Headache was found correlated with the parameters of sleep quality in this population. Further, pain in the head, neck, back, lower back, and shoulder were correlated with sleep latency.

There is evidence that stress, sleep disturbances, anxiety, and depression are a common reactions to the pandemic $[17,37,38]$. And poor sleep has been shown, in the lit- 
erature, to be another factor in causing increasing chronic pain [17]. In addition, this study showed that individuals' depression scores were related to the parameters of sleep quality during COVID-19 in Turkey. Especially, subjective sleep quality, sleep disturbances, daytime dysfunction, and total score were moderately correlated with depression scores. As expected, the pain in all body parts has shown a correlation with depression scores in the study.

The biopsychosocial dimension of pain may help to explain the relationship that musculoskeletal pain, sleep quality, and depression have shown during the pandemic. It is known that lack of sleep is a cause of tension headache [39]. There were studies that try to explain which one causes the other, sleep disturbances or headache. We know these circumstances may affect each other, and also that depression may occur with sleep disturbance or chronic headache $[39,40]$. In cases of depression, chronic pain, and sleep disturbance, it was shown that abnormal patterns of limbic activation occur in the brain. And this increasing activation causes emotional responses, increasing the emotional component of pain and producing shallower sleep in individuals [41]. These neurobiological processes may also have accompanying psychosocial processes in individuals during the pandemic, and it may cause a vicious circle. Pfefferbaum and North [42] mentioned that the COVID-19 pandemic has alarming effects on emotional and social functioning for individuals and on public health. This study showed that pain, sleep disturbance, and depression negatively affect mental and physical health in Turkey similar to other countries during the pandemic. And we cannot know which one of these factors most affects another, but programs may be developed to improve public health in response to the effects of the pandemic.

A limitation of the study is that because the ongoing pandemic processes, the evaluations of the study could not be made face to face. In addition, another limitation of this study is that the study could not performed after the conclusion of the pandemic because the pandemic still continues in Turkey and worldwide. If this were done, the comparisons would have been more informative and valuable.

While some studies have examined musculoskeletal pain, sleep quality, and depression in individuals during the COVID-19 lockdown, no previous research considers all of them and their associations with each other in one study. In addition, we think that this study is valuable for having been carried out with a large number of individuals and for investigating pain profiles during the pandemic in Turkey's population. The findings of this study showed that the profile of musculoskeletal pain was differentiated in different body parts, intensities, and age groups in Turkey during the pandemic. Sleep quality is associated with musculoskeletal pain in various body parts and with depression. Further, musculoskeletal pain in these body parts is correlated with depression during the COVID-19 lockdown in individuals in Turkey. More studies are needed that shed light on the factors that cause musculoskeletal pain and the relationship between musculoskeletal pain, sleep parameters, and depression during the pandemic. Also, the effects of the pandemic must be investigated after the pandemic and programs developed to improve these effects.

\section{CONFLICT OF INTEREST}

No potential conflict of interest relevant to this article was reported.

\section{FUNDING}

No funding to declare.

\section{ORCID}

Merve Karatel, https://orcid.org/0000-0003-4837-2359

Zeynep Irem Bulut, https://orcid.org/0000-0002-3547-1350

Erkin Oguz Sari, https://orcid.org/0000-0001-7770-4581

Zerrin Pelin, https://orcid.org/0000-0002-8841-0068

Yavuz Yakut, https://orcid.org/0000-0001-9363-0869

\section{REFERENCES}

1. World Health Organization. WHO coronavirus (COVID-19) dashboard [Internet]. Geneva: World Health Organization; 2021. Available at: https://covid19.who.int/.

2. Fowler JH, Hill SJ, Levin R, Obradovich N. Stay-at-home orders associate with subsequent decreases in COVID-19 cases and fatalities in the United States. PLoS One 2021; 16: e0248849.

3. Zhou X, Snoswell CL, Harding LE, Bambling M, Edirippulige $\mathrm{S}$, Bai X, et al. The role of telehealth in reducing the mental health burden from COVID-19. Telemed J E Health 2020; 26: 377-9.

4. Majumdar P, Biswas A, Sahu S. COVID-19 pandemic and lockdown: cause of sleep disruption, depression, somatic pain, and increased screen exposure of office workers and students of India. Chronobiol Int 2020; 37: 1191-200.

5. Vedaa Ø, Erevik EK, Hysing M, Hayley AC, Sivertsen B. Insomnia, sleep duration and academic performance: a na- 
tional survey of Norwegian college and university students. Sleep Med X 2019; 1: 100005.

6. Toprak Celenay S, Karaaslan Y, Mete O, Ozer Kaya D. Coronaphobia, musculoskeletal pain, and sleep quality in stay-at home and continued-working persons during the 3-month Covid-19 pandemic lockdown in Turkey. Chronobiol Int 2020; 37: 1778-85.

7. Finan PH, Goodin BR, Smith MT. The association of sleep and pain: an update and a path forward. J Pain 2013; 14: 1539-52.

8. Haack M, Simpson N, Sethna N, Kaur S, Mullington J. Sleep deficiency and chronic pain: potential underlying mechanisms and clinical implications. Neuropsychopharmacology 2020; 45: 205-16.

9. Rodríguez-Nogueira Ó, Leirós-Rodríguez R, Benítez-Andrades JA, Álvarez-Álvarez MJ, Marqués-Sánchez P, PintoCarral A. Musculoskeletal pain and teleworking in times of the COVID-19: analysis of the impact on the workers at two Spanish universities. Int J Environ Res Public Health 2021; 18: 31.

10. Mucci F, Mucci N, Diolaiuti F. Lockdown and isolation: psychological aspects of COVID-19 pandemic in the general population. Clin Neuropsychiatry 2020; 17: 63-4.

11. Gualano B, Bonfa E, Pereira RMR, Silva CA. Physical activity for paediatric rheumatic diseases: standing up against old paradigms. Nat Rev Rheumatol 2017; 13: 368-79.

12. Lippi G, Henry BM, Bovo C, Sanchis-Gomar F. Health risks and potential remedies during prolonged lockdowns for coronavirus disease 2019 (COVID-19). Diagnosis (Berl) 2020; 7: 85-90.

13. Song J, Dunlop DD, Semanik PA, Chang AH, Lee YC, Gilbert $\mathrm{AL}$, et al. Reallocating time spent in sleep, sedentary behavior and physical activity and its association with pain: a pilot sleep study from the Osteoarthritis Initiative. Osteoarthritis Cartilage 2018; 26: 1595-603.

14. Wang C, Pan R, Wan X, Tan Y, Xu L, Ho CS, et al. Immediate psychological responses and associated factors during the initial stage of the 2019 coronavirus disease (COVID-19) epidemic among the general population in China. Int J Environ Res Public Health 2020; 17: 1729.

15. Šagát P, Bartík P, Prieto González P, Tohănean DI, Knjaz D. Impact of COVID-19quarantine on low back pain intensity, prevalence, and associated risk factors among adult citizens residing in Riyadh (Saudi Arabia): a cross-sectional study. Int J Environ Res Public Health 2020; 17: 7302.

16. Fallon N, Brown C, Twiddy H, Brian E, Frank B, Nurmikko T, et al. Adverse effects of COVID-19-related lockdown on pain, physical activity and psychological well-being in people with chronic pain. Br J Pain 2021; 15: 357-68.

17. Clauw DJ, Häuser W, Cohen SP, Fitzcharles MA. Considering the potential for an increase in chronic pain after the $\mathrm{CO}$ VID-19 pandemic. Pain 2020; 161: 1694-7.
18. Hruschak V, Flowers KM, Azizoddin DR, Jamison RN, Edwards RR, Schreiber KL. Cross-sectional study of psychosocial and pain-related variables among patients with chronic pain during a time of social distancing imposed by the coronavirus disease 2019 pandemic. Pain 2021; 162: 619-29.

19. Jackson T, Wang Y, Wang Y, Fan H. Self-efficacy and chronic pain outcomes: a meta-analytic review. J Pain 2014; 15: 800 14.

20. Burke AL, Mathias JL, Denson LA. Psychological functioning of people living with chronic pain: a meta-analytic review. Br J Clin Psychol 2015; 54: 345-60.

21. Wang CC, Chao JK, Chang YH, Chou CL, Kao CL. Care for patients with musculoskeletal pain during the COVID-19 pandemic: physical therapy and rehabilitation suggestions for pain management. J Chin Med Assoc 2020; 83: 822-4.

22. Beck AT, Ward CH, Mendelson M, Mock J, Erbaugh J. An inventory for measuring depression. Arch Gen Psychiatry 1961; 4: 561-71.

23. Ağargün MY, Kara H, Anlar Ö. The validity and reliability of the Pittsburgh Sleep Quality Index. Turk Psikiyatri Derg 1996; 7: 107-15.

24. Buysse DJ, Reynolds CF 3rd, Monk TH, Berman SR, Kupfer DJ. The Pittsburgh Sleep Quality Index: a new instrument for psychiatric practice and research. Psychiatry Res 1989; 28: 193-213.

25. Boonstra AM, Schiphorst Preuper HR, Balk GA, Stewart RE. Cut-off points for mild, moderate, and severe pain on the visual analogue scale for pain in patients with chronic musculoskeletal pain. Pain 2014; 155: 2545-50.

26. Taylor R. Interpretation of the correlation coefficient: a basic review. J Diagn Med Sonogr 1990; 6: 35-9.

27. Amro A, Albakry S, Jaradat M, Khaleel M, Kharroubi T, Dabbas A, et al. Musculoskeletal disorders and association with social media use among university students at the quarantine time of COVID-19 outbreak. J Phys Med Rehabil Stud 2020; 1: 105.

28. Bontrup C, Taylor WR, Fliesser M, Visscher R, Green T, Wippert PM, et al. Low back pain and its relationship with sitting behaviour among sedentary office workers. Appl Ergon 2019; 81: 102894.

29. Köseoğlu Toksoy C, Demirbaş H, Bozkurt E, Acar H, Türk Börü Ü. Headache related to mask use of healthcare workers in COVID-19 pandemic. Korean J Pain 2021; 34: 241-5.

30. Shanthanna H, Strand NH, Provenzano DA, Lobo CA, Eldabe $\mathrm{S}$, Bhatia A, et al. Caring for patients with pain during the COVID-19 pandemic: consensus recommendations from an international expert panel. Anaesthesia 2020; 75: 935-44.

31. Murray CJ, Atkinson C, Bhalla K, Birbeck G, Burstein R, Chou $\mathrm{D}$, et al. The state of US health, 1990-2010: burden of diseases, injuries, and risk factors. JAMA 2013; 310: 591-606.

32. Nicholson RA, Houle TT, Rhudy JL, Norton PJ. Psychological risk factors in headache. Headache 2007; 47: 413-26. 
33. Grover S, Sahoo S, Mehra A, Avasthi A, Tripathi A, Subramanyan A, et al. Psychological impact of COVID-19 lockdown: an online survey from India. Indian J Psychiatry 2020; 62: 354-62.

34. Rossi R, Socci V, Talevi D, Mensi S, Niolu C, Pacitti F, et al. COVID-19 pandemic and lockdown measures impact on mental health among the general population in Italy. Front Psychiatry 2020; 11: 790.

35. Gupta AK, Sahoo S, Mehra A, Grover S. Psychological impact of 'Lockdown' due to COVID-19 pandemic in Nepal: an online survey. Asian J Psychiatr 2020; 54: 102243.

36. Linton SJ. A review of psychological risk factors in back and neck pain. Spine (Phila Pa 1976) 2000; 25: 1148-56.

37. Wang J, Gong Y, Chen Z, Wu J, Feng J, Yan S, et al. Sleep disturbances among Chinese residents during the Coronavirus Disease 2019 outbreak and associated factors. Sleep Med
2020; 74: 199-203.

38. Cellini N, Canale N, Mioni G, Costa S. Changes in sleep pattern, sense of time and digital media use during COVID-19 lockdown in Italy. J Sleep Res 2020; 29: e13074.

39. Paiva T, Batista A, Martins P, Martins A. The relationship between headaches and sleep disturbances. Headache 1995; 35: 590-6.

40. Rains JC, Poceta JS. Headache and sleep disorders: review and clinical implications for headache management. Headache 2006; 46: 1344-63.

41. Boakye PA, Olechowski C, Rashiq S, Verrier MJ, Kerr B, Witmans $\mathrm{M}$, et al. A critical review of neurobiological factors involved in the interactions between chronic pain, depression, and sleep disruption. Clin J Pain 2016; 32: 327-36.

42. Pfefferbaum B, North CS. Mental health and the Covid-19 pandemic. N Engl J Med 2020; 383: 510-2. 